and reaccreditation in the future. ${ }^{9}$ The authority will need to involve local consultants and general practitioners from outside the district in audit and reaccreditation.

\section{PRIMARY CARE PROVIDER UNIT}

This unit or trust will be formed from the provider arms of the current community unit and family health services authority. The unit will contract for community services with the purchasing authority or general practice executive committee team or with individual practices or the private sector. Some competition for the provision of primary care and community services will therefore be introduced.

\section{PRIMARY HEALTH CARE TEAMS}

General practitioners and primary health care teams will negotiate a contract with the purchaser and provide services under the contract. Not all practices will want to contract for an expanded range of services and local circumstances will also affect contracting decisions. The purchaser may contract with the primary care provider unit instead of primary care teams. Primary care teams will manage their own budget for staff, prescribing, general medical services, etc, and be accountable to the purchaser for their performance.

The model envisages that a regional management tier will have an important role in strategy development, monitoring, and accountability. ${ }^{10}$

\section{Consequences of the model}

The model will give general practitioners the enhanced influence, powers, and responsibilities of fundholders without the administrative burden. They will have a flexible local contract focusing on the needs of their patients. Doctors elected to the general practice executive committee will have additional purchasing responsibilities.

Flexible contracting will increase accountability to management and make management sensitive to the needs of patients. Integration of primary and secondary care is central to an internal market driven by primary care and should encourage a needs led rather than a service dominated approach.

Under our model contracting may cause difficulties with dispensing, deputising, partnership agreements, profitability, and investment in premises. Arbitration will be needed to prevent exploitation by the purchasing authority. The national contract has had many successes and there is a danger these could be threatened by fragmentation.

The model requires that general practitioners and managers develop new skills particularly in contracting. The evidence that contracting is an efficient mechanism in the NHS is still limited. General practitioners will be concerned that purchasing pressures will disturb the doctor-patient relationship, and doctors' perception of their role as the patient's friend and ally.

\section{Conclusions}

Our model is one possible means of enhancing the benefits of the NHS reforms while minimising some of the problems of fragmentation. The model gives a clear role to general practitioners, reduces their administrative burden, and will stimulate the development of primary care services. A strong contracting framework will be coordinated right across the NHS, balancing the increased responsibilities and power of general practitioners and their accountability for the outcome of the care they provide.

1 Tudor Hart J. The inverse care law. Lancet 1971;i:405-12

2 Department of Health. Caring for people. London: HMSO, 1989. (Cmnd 849.) O'Dowd TC, Wilson AD. The future of general practice: set menus and clinical freedom. BMf 1991;303:450-2.

Taylor D. Developing primary care: opportunities for the 1990s. London: King's Fund Institute, 1991.

5 Glennerster H, Matsagnis M, Owen P. A foothold for fundholding. London: King's Fund Institute, 1992

6 Foster A. FHSAs: today's and tomorrow's priorities. London: Department of Health, NHSME, 1991.

7 Bosanquet N. The future of general practice: family doctors and payment systems: the local option. $B M$ I $^{1991 ; 303: 233-4 .}$

8 Roland $M$. The future of general practice: fundholding and cash limits in primary care: blight or blessing. $B M \mathcal{F}$ 1991;303:171-2.

9 Irvine D. Managing for quality in general practice. London: King's Fund Institute, 1990.

10 North East Thames Regional Health Authority. Primary health care in the 90s-a strategic statement. London: North East Thames Regional Health Authority, 1991

(Accepted 1 December 1992)

\title{
The Future of FHSAs
}

\section{FHSAs and prescribing}

\author{
Rhona Panton
}

This is the fifth and final article in a series of articles on the future of family health services authorities

Department of Pharmacy Policy and Practice, Keele University,

Keele ST5 5BG

Rhona Panton, head of

department

Series editor:

Dr Andrew Harris

BMF 1993;306:310-4
Prescribing by general practitioners cost the NHS $£ 2 \cdot 3$ billion in 1991 - the biggest single cost after staffingand the drug bill is now rising at $11 \%$ a year. Family health services authorities and their professional advisers are charged with the challenging task of "improving the quality and cost effectiveness of prescribing," but any consideration of prescribing costs requires an understanding of how they are affected by factors outside the family health services authority's control. ${ }^{\prime}$ These include price regulation by the government; differential pricing between hospitals and the community; the use of generic and branded products; and the prescription charge, which conceals from the general public the true cost of medicines.

\section{Containing costs}

In most developed countries the pharmaceutical industry is an important part of the economy and its products contribute directly to health gain. Governments, which are purchasers, are faced with the dilemma of safeguarding the benefits while containing the price of drugs. The ability to develop new drugs is now greater than most countries' ability to pay for them without rationing or screening systems-for example, France controls individual prices and Germany has initiated a reference system which pays a basic price for all drugs in the same category. ${ }^{2}$ In Great Britain the government's control system is the prescription pricing regulation scheme, a voluntary agreement between the industry and the government that agrees profitability on NHS business. A measure of the scheme's effectiveness is that the United Kingdom has the fifth highest drug prices in Europe (about where we should expect to be). ${ }^{3}$ The industry recognises the pressures to contain costs and knows that it must concentrate on developing clearly better products, work towards European standardisation of prices, and 
FHSAs have an important role in improving the standards of prescribing and dispensing

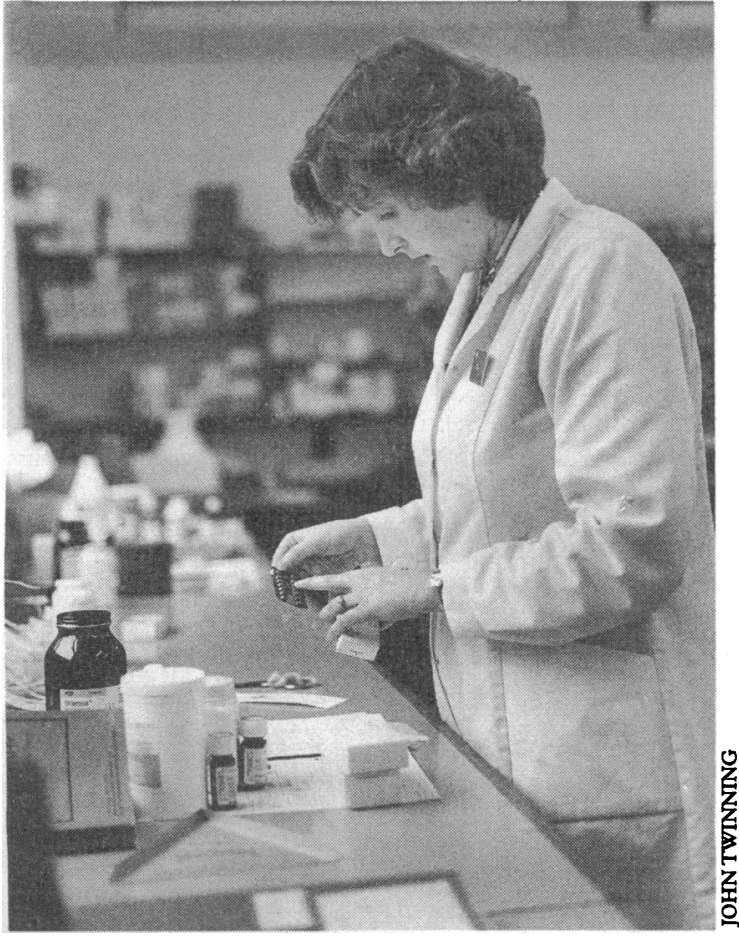

accept the generic market in return for an extended patent period. ${ }^{4}$

Hospitals are given large discounts on drugs to encourage consultants to use and endorse them. Such discounts can mean the price is many times lower for the branded drug than for the generic drug and consultants may be unaware of the price in the community. The good business outcome of this policy for the pharmaceutical industry is evident in a recent survey in which four out of five general practitioners claimed to follow the hospital prescription on patient discharge. ${ }^{5}$ Drugs are expensive in primary care, not normally because of the unit cost, but because many patients take them for many years. Thus hospital recommendations, based on discounted prices, can result in high cost commitments for primary care.

Generic substitution is common in hospitals, but only two out of five prescriptions are written generically in primary care. ${ }^{6}$ The percentage of generic prescribing could be increased if legislation allowed the prescriber to "tick the box" for the generic equivalent or, as in North America, the pharmacist could, with proper safeguards, offer the generic drug as an option. An incentive in the United Kingdom could be to reduce the prescription charge by, say, $50 \mathrm{p}$ if the patient selected the generic option.

Most European countries have a copayment system for medicines, but in the United Kingdom patients are unaware of drug costs and only $22 \%$ pay prescription charges. Ways of informing them would be to put the price on the pharmacist's label and make financial need the only basis for exemption from prescription charges: more patients would pay the charges (which could have an annual ceiling) but the unit payment could be reduced.

\section{Improving prescribing}

None the less real improvements could be made in prescribing, and fundholders have shown savings. Drugs are a science based industry's problem solving approach to health care and should be seen in that context. Prescribing is one possible treatment option; others include counselling, educating patients on self limiting illnesses, and changes in lifestyle to improve health. Better definition of expected health gain, treatment goals, and outcome measures for drug treatment would reduce unnecessary prescribing; provide safer treatment; and ensure that drugs are used appropriately. Family health services authorities have a key role in helping general practitioners to achieve these outcomes.

All family health services authorities are funded equally to purchase professional advice on prescribing, but evaluation of the success of medical advisers has been impeded by a variety of changes that have affected prescribing. These include specialist drugs, such as erythropoietin and growth hormone, being moved from hospital to primary care, mostly because hospitals are cash limited and primary care budgets are not. Health promotion clinics are diagnosing and treating disease-sometimes with drugs. Finally, doctors planning to become fundholders may not have worked assiduously to control prescribing in the knowledge that their budgets will be based on historical costs. The outcome of these changes is that the drug bill is rising faster than it has for some years and above the rate of inflation.

Three models of good practice have emerged. In the first, medical advisers with a reasonable workload have developed good relationships with practices and are now well placed to advise on prescribing. In the second, medical advisers have acted as diagnosticians to determine the needs and concerns of each practice and to identify those where prescribing is an issue; pharmaceutical advisers then visit and offer help. In the third, medical advisers have developed a management role in the family health services authority, addressed wider health strategy, and planned practice development; pharmaceutical advisers have led in all aspects of prescribing, involving the medical adviser only when the issue is whether to prescribe rather than what to prescribe. The NHS Management Executive has now created a prescribing team, one of whose first actions has been to require that prescribing is a priority for medical advisers. This has required some family health services authorities to reconsider wider roles, to focus medical advice on prescribing, and to agree targeted savings.

Prescribing analysis and cost data are a computer record of all general practitioner prescribing and provide an excellent research base to investigate changes in prescribing, trends, and the health gain and cost implications. As data are available on individual drugs and the doctor ordering them, professional advisers can use them to inform their visits to apparent high and low cost prescribers and to make recommendations for change. Key ways in which they have worked to lower costs are in reducing the prescribing rate (which is often high in poorer areas); increasing generic prescribing by informing people of the savings, addressing concerns, and, in the west midlands, offering laboratory analysis if patients believe there to be a difference in symptom control; and providing draft formularies and guidance on their management.

"Underprescribers" may be simply prudent prescribers but may also not be informed on the latest thinking on the benefits of drug treatment. They can be helped to improve the quality of their prescribing by drug treatment protocols, formularies including newer drugs, and targeted drug information.

\section{Prescribing at the interface}

Professional advisers can help general practitioners to determine their requirements for prescribing at the primary and secondary care interface and ensure that purchasers include these criteria in contracts. These requirements include the development, jointly with hospital consultants, of drug treatment protocols for consistency of clinical management, specific policiesfor example, on the use of antibiotics - and shared care 
protocols for new drugs they are asked to prescribe. Professional advisers who are members of drug and therapeutic committees can ensure that primary care concerns are heard and addressed and that they are informed of any changes in hospital prescribing that affect them. A development of this will be to agree appropriate drug treatment when patients are in hospital to ensure we do not recreate the difference in drug policies shown between United States acute care and veterans hospitals.

Problems in prescribing at the interface present a strong case for commissioning agencies that would consider drug purchasing across both care sectors. Meanwhile professional advisers, both directly and through their managers, address changes that affect prescribing in primary care with regional health authorities, purchasers, and providers; among these changes will be better information systems between secondary and primary care so that patients being discharged into the community have their medicines available and that "at risk" patients are identified: pharmaceutical services must follow the patient.

Evaluation of the cost effectiveness of drug treatment is in its infancy, and health economics can inform the debate. General practitioners need such information in a more relevant form, related to current patient needs and gains. Family health services authority advisers are well placed to develop this research area, to evaluate drug use, and to facilitate local work by general practitioners on targeted areas.

\section{Prescribing in general practice}

All non-fundholding practices have indicative prescribing amounts set and monitored by professional advisers. The amounts are primarily derived from historical practice data, with new weightings for age and sex and a formula that includes an "uplift factor." The system to date has lost credibility among general practitioners since the uplift factors proved inaccurate and the predicted outturns erratic. The latest development is that each region has a prescribing budget for all prescribers, which will require development in a meaningful way with prescribing norms.

Practice specific work includes analysis of prescribing and visits to understand the reasons for apparent over- and under-prescribing. Prescribing analysis and cost data offer valuable insights into practice indicators of prescribing that are worthy of further investigation (box).

Regional drug information centres inform professional advisers on new drugs and their likely cost implications. They also provide unbiased information on how new products compare with the range, give cost comparison charts, and draft shared care protocols for development and agreement with consultants and general practitioners.

Practice visits have resulted in identifying training needs, the need for (and writing of) policies to improve the quality of prescribing, and the development of drug use analysis, including all of the cost implications.

\section{Will budgets be cash limited?}

Fundholders already have cash limited budgets. In the west midlands 26 fundholders made a combined saving of over $£ 1 \mathrm{~m}$ on their drug bill: the ability to retain the savings for practice development seems to have provided a strong incentive. Early assessments indicate that fundholding has been a success in enabling practices to manage their budgets and develop patient services in the way it chooses. If confirmed, these outcomes are likely to make fundholding the norm provided that the Treasury continues to allow health ministers this method of resource management. Since

\section{Some indicators of prescribing in a} practice

- How actively is a disease-for example, asthmabeing treated?

- How do practices with specific expertise-for example, teaching practices and those where doctors have hospital sessions-compare with others?

- How do fundholders and non-fundholding general practitioners differ?

- How do consultation and prescribing rates relate to measures of deprivation and practice population compared with similar practices?

- What are the priority training areas and how can they be met?

- Which doctors are most or least likely to use new products?

- Which outdated drugs are still used?

- In which therapeutic areas would a protocol help?

- How effective is the intervention of family health services authority professional advisers measured by reanalysis of prescribing analysis and cost data?

the general election the move to fundholding has been dramatic and the suggestion that cash limiting will apply to all practitioners will fuel this trend. It is, of course, too early to judge whether fundholding has improved the quality of prescribing, and research on this is urgently needed.

More practices might consider a prescribing contract alone if they were allowed to retain a percentage of the savings. These could be monitored against referral rates if each family health services authority had a minimum contract dataset (all of the necessary contract data) for all patients. Another management system could be to calculate the modest savings achievable by practices or locally based groups of practices and to pay this sum to those who achieve the target. Finally, treatment protocols could each be delivered as an item of service-a logical step from payment for health promotion clinics which diagnose disease. All would have to allow agreed improved prescribing costs to be added. Local incentive schemes are the most likely way of getting general practitioners to change prescribing habits by ensuring that they see the benefits retained in local health care. Until then, talk of cash limited budgets will only add to general practitioners' sense of being required to make too many changes too quickly.

Will expensive specialist prescribing require health rationing?

Health economists regularly forecast rationing of expensive drugs: an easy target since their costs-and the patients receiving them-are readily identifiable. "Shared care" high cost drugs are, however, a very small part of the primary care drug bill-less than $1 \%$ in the west midlands in 1991. All high cost drugs are already rationed in hospitals through drug and therapeutics committees and clinical pharmacy services. Best practice screening before a drug is used includes:

- Appraisal of current prescribing practice and identification of the likely benefits, and of the risk in not introducing the product

- An estimate of the cost to the hospital and identification, if appropriate, of authorised prescribers

- A review period and reassessment of the benefits. ${ }^{7}$

Such screening ensures appropriate use of expensive medicines and is a model of the way in which any NHS development should be evaluated before being implemented. Purchasers will seek such systems from providers when setting contracts. Family health services authority professional advisers will be keen to see 
that the system is not bypassed by hospital consultants who, having had a request to use a product turned down, ask general practitioners to initiate the prescription.

When consultants ask general practitioners to share the care-that is, to prescribe specialist productsthey accept an obligation to inform: shared care protocols should explain the rationale of treatment, cost, side effects, and the aspects of care for which the hospital consultant will remain responsible. Many shared care products are high cost. Fundholders, if not reimbursed for their use, may insist that drugs be included in the hospital price. This will result in a two tier system, where patients will be treated (by nonfundholders) or not. The logical way forward is for purchasers to agree the number of patients they will pay for and for the region to ensure that this provision is adequate. The cost will then follow the patient. Further rationing of high cost drugs should be resisted until the costs of other developments are equally well known, debated among peer groups, and evaluated in use.

Of more urgent concern is the uncontrolled way in which new drugs, formulations, and presentations (not of individual high cost but likely to be used for large numbers of patients) are introduced, without assessment of health gain or cost, into primary care prescribing by consultants' recommendations, patient pressure, general practitioners' individual decisions. Such assessments should be nationally obtained from research units and available to all prescribers. Consultants and general practitioners need clearer explanations that a resource can be used only once, and higher expenditure on prescribing in primary care means loss of development money for the NHS. General practitioners need assurance that control of expenditure will result in the savings being retained in local health care. Incentive schemes (previously described) with agreed local benefits, such as the provision of more health visitors, are most likely to reassure general practitioners on this point. The development of purchasers must include a requirement that providers examine the appropriateness of all prescribing (including the price differential of products in secondary and primary care) and that discharge and outpatient recommendations are for a therapeutic group, rather than specific products. While professional advisers can, and do, work towards "once only" savings such as changes in the prescribing of generic drugs, the real control needed is the introduction of new products only after assessment of the health gain and financial consequences.

On the other hand, a harder look should be taken at the range of prescribable products of doubtful value, such as, peripheral and central vasodilators, cough mixtures, baby creams, mild analgesics, and shampoos, and the value of others - for example, vaccination for foreign travel ${ }^{8}$ - should be reassessed. The products alone cost many millions, to which the cost of professionals' time should be added. General practitioners can be supported in refusing requests for these products in two ways: by the extension of the limited list (planned for June 1993) and by a structured patient education campaign to inform patients that the doctor cannot prescribe all medicines and that some illnesses are self limiting.

\section{Prescribing and dispensing standards}

Professional audit, established in medical practice and proposed for pharmacists, ${ }^{9}$ is invaluable for prescribing analysis and offers the opportunity to set treatment goals, measure success in achieving them, and re-evaluate the process. Practice indicators from prescribing analysis and cost data can illumine audit and highlight discussion areas (box).
Further research is needed to understand the reasons why doctors prescribe as they do, and the cultural needs of the society in which the doctor works, including issues of deprivation and doctor and patient expectation, must be addressed before any judgment of excessive prescribing is made. ${ }^{10}$ The legal mechanism for determining that judgment comprises a committee of three doctors, appointed by the family health services authority (with specific criteria for their selection). A judgment of excessive prescribing can result in an amount being deducted from the doctor's remuneration. ${ }^{11}$ This procedure is seen by most family health services authorities as a last resort, after practices deemed to be prescribing inappropriately have been offered information and help to lower their prescribing rates and costs. Some practitioners, especially singlehanded ones, may benefit from training initiatives for doctors to reduce "sympathetic prescribing" under pressure from patients.

Pharmacists' dispensing standards are tested by the family health services authority and by the Royal Pharmaceutical Society of Great Britain, whose mandatory code of ethics includes standards of practice for dispensing. Disciplinary action is taken against any pharmacist who fails to comply and the royal society's inspectors, in their role as enforcers of both the Medicines Act and the profession's code of standards, visit community pharmacists regularly. Collaboration between the royal society and family health services authorities would be a sensible way forward in improving dispensing standards.

\section{Future roles for community pharmacists}

Community pharmacy, an underused resource, will change with the NHS reforms and become better integrated into the prescribing process to ensure optimum pharmaceutical care. In hospitals pharmacists already advise prescribers, through drug information and clinical pharmacy services, and encourage cost effectiveness and patient safety in drug usage. In primary care prescribers rightly expect a safe and accurate dispensing service, but they have lost the regular advisory service and are most often given drug information by drug company representatives. Occasional visits from medical and pharmaceutical advisers need to be supplemented by a readily available and local source of advice and information. One option would be for the family health services authority to employ community pharmacists on a sessional basis to visit local practices. ${ }^{12}$ The authority's professional advisers could target an area of concern, brief community pharmacists on the issues, and use their practice visits to inform general practitioners. Such training could be funded by the postgraduate training allowance.

More effective use could be made of the pharmacy's patient medication records - for example, to analyse a practice's prescribing patterns and make recommendations for change. Patients whose ability to take

\section{Some indicators useful for audit of prescribing in general practice}

- Treatment consensus among partners and consistency of prescribing

- High cost areas where drug information can help with comparative costs

- The need for a repeat prescribing system; a list of drugs preferred by the practice and practice policiesfor example, on prescribing antibiotics and benzodiazepines

- Comparison of practice prescribing with the family health services authority average 
medicines is a critical issue could be encouraged to use the same community pharmacy on each occasion. The pharmacist could be informed of the risk and would be well placed to help the prescribers achieve their therapeutic objectives (for example, by offering compliance aids, visiting the home, or checking usage of the product) and to inform them of any problems.

Medicines do not come without risks, and patients are harmed by them through no fault of the drug or the prescriber. Pharmacists should lead in actively encouraging patients to report side effects, or lack of treatment success, and either resolve the problem or inform the prescriber.

Fundholding strengthens the case for pharmacists working in health centres. ${ }^{13}$ In addition to dispensing, community pharmacists could offer the same range of services as their hospital counterparts: they could screen and recommend new products to prescribers, analyse prescribing patterns, and ensure safe and appropriate drug therapy since they would have access to patients' notes.

Family health services authorities and consumers will, however, wish to ensure the viability of local pharmacies, with their convenience of access, the savings pharmacists make to the NHS by responding to common ailments, and new services such as needle exchange programmes for drug misusers. Their future would best be secured by implementing the repeat dispensing system, recommended in a recent Department of Health report, ${ }^{9}$ and developing a contract that pays for services other than dispensing - for example, health promotion-where the pharmacist can offer opportunistic advice without the need for identification or referral.

A reduction in the number of single handed pharmacies is likely, as is the development of specialist pharmaceutical services (such as parenteral nutrition) previously available only in hospitals. Joint treatment protocols should be extended to the community pharmacy wherever possible so that local practices know what the pharmacist will sell. Such protocols will be essential with the extension of the limited list-indeed, fundholders may find reimbursing pharmacists, working to a protocol, cheaper than writing prescriptions.

\section{Conclusion}

The quality of prescribing can be improved by the activities of family health services authority professional advisers. Some outcomes, such as reducing unnecessary prescribing, will result in savings which could be maximised if family health services authorities were empowered either to make fundholding the norm or to offer incentives to all general practitioners. Certain quality developments will have little or no cost saving effect but will improve patient safety-for example, the preferential use, wherever possible, of well tried drugs with a long safety record. Others, such as the use of newer drugs when these are appropriate to the patient's needs, will cost more.

The future roles of medical and pharmaceutical advisers are unclear. Purchasers will need more advice on prescribing; in a contract setting this will include the definition of health gain from drugs, secondary care agreement of joint treatment protocols, systems to ensure appropriate prescribing at the primary care and secondary care interface, and definition of the

\section{Summary}

- Drug costs are rising worldwide and price controls are set by governments

- The United Kingdom has a unique database of prescribing from which practice indicators and audit of prescribing can be derived

- FHSAs are funded to provide professional advice to prescribers

- Most fundholders have achieved savings on their drug bills; other incentive schemes are needed

- Research is needed to measure prescribing outcomes and health gain before new drugs and formulations are used

- There are prescribing interface issues between primary and secondary care which may best be resolved by the development of purchasers

- Community pharmacists have a developing role to play in improving prescribing

standards and range of pharmaceutical services. These professional advisers will need statistical and analytical skills as well as expertise in drug therapy. The family health services authority's role of advising general practitioners, both on improving their prescribing and on how to ensure value for money, will remain and they will be required to show the effectiveness of their interventions. A golden opportunity and the enthusiasm of professional advisers would be lost if cost reduction was required to dominate without full analysis of the health gain to be achieved from improved (and sometimes costlier) drug treatment. Increasing links between family health services authorities and postgraduate education and academic units are important and enable commissioning input to training and research agendas.

Drugs will continue to have a key role in preventing and treating disease and in ameliorating symptoms. These health gains keep people independent, employed, and out of hospital, but their cost effectiveness will need to be continually evaluated alongside other options.

1 Department of Health. Improving prescribing-working for patients. London: $\mathrm{DoH}, 1990$.

2 National Consumer Council. Pharmaceuticals-a consumer prescription. London: NCC, 1991.

3 Remit Consultants. Impediments to parallel trade in pharmaceuticals within the European Community. London: HMSO, 1992. (Final report prepared for DG IV of the Commission of the European Communities.)

4 Kessler A. International solutions needed for drug marketings. Chemist ana Druggist 1992;236:605.

5 School of Management, University of Manchester Institute of Science and Technology. Determining the drug information needs of general practitioners. Liverpool: Medicines Resource Centre, 1992.

6 Department of Health. Statistical bulletin on general pharmaceutical services, England and Wales. Government Statistical Service 1980-1991. London: $\mathrm{DoH}, 1992$.

7 Baker John A Lant, Ariel F, Sutters A. Seventeen years' experience of a voluntarily based drug rationalisation programme in hospital. $B M \mathcal{F}^{\prime} 1988$; 297:465-9.

8 Typhoid vaccination. Weighing the options [Editorial]. Lancet 1992;340: $341-2$

9 Royal Pharmaceutical Society of Great Britain. Pharmaceutical care: the future for community pharmacy. Report of the joint working party on the future role of the community pharmaceutical services. London: RPSGB, 1992.

10 Bradley C. Uncomfortable prescribing decisions: a critical incident study $B M 7$ 1992;304:294-6.

11 NHS Management Executive. Excessive prescribing by GPs; referral to a professional committee. London: NHSME, 1992. (EL(92)90.)

12 Newton-Syms FAO, Dawson PH, Calvert RT, Cook J, Feeley M, Booth TG. The influence of a pharmacist on general practitioner prescribing. Pharmaceutical fournal 1988;294:R2.

13 Parr C. Equal partnerships in primary health care teams. College of Pharmacy Practice Annual Lecture. Pharmaceutical fournal 1992;248:616. 